\title{
Cartesian product and Topology On Fuzzy BI-Algebras
}

\author{
Gerima Tefera , Abdi Oli \\ Wollo University, College of Natural Science, Department of Mathematics, \\ Dessie, Ethiopia
}

\begin{abstract}
In this paper,the concepts of homomorphism in fuzzy BI-algebra is introduced, and also basic properties of homomorphisms are investigated.

The cartesian product in fuzzy ideals of BI-algebra is investigated with related properties; The concepts of fuzzy topology on BIalgebra elaborated.
\end{abstract}

Keywords- Implication algebra,BAlgebra,and BCK- Algebra.

\section{INTRODUCTION}

$\mathbf{E}$ VER Imai,Y. and Iseki,K. in [9] introduced two classes of abstract algebras, BCK-Algebras and BCI- algebras. Neggers,J. and Kim,H.S.in 14. initiated the idea of B-algebras which is a generalization of BCK-algebras and they also introduced the notion of d- algebras which is another useful generalization of BCK -algebras and they investigated different relations between d-algebras and BCK-algebras in [14.

Jun,Y.B., Roh,E.H., and Kim,H.S. in [10] investigated on $\mathrm{BH}-$ algebras which are a generalization of $B C K / B C I / B$ - algebras. Borzooel and et al in 2] introduced the notion of implicative BCK-algebras and also discussed that implication algebras are equivalent to the dual implicative BCK-algebras; and Huang in 7 define an algebra $(X, \star, 0)$ of type $(2,0)$ is called a BCI-algebra if for any $a, b, c \in X$ the following holds:

1) $((a \star b) \star(a \star c)) \star(c \star b)=0$.

2) $(a \star(a \star b)) \star b=0$.

3) $a \star a=0$.

4) $a \star b=0$ and $b \star a=0$ imply $a=b$.

If a BCI- algebra $\mathrm{X}$ satisfies the property $0 \star a=$ 0 , then $(X, \star, 0)$ is called a BCK-algebra.

Arsham Borumand Saeid and et al in 1 in- troduced $B I$-Algebra as a generalization of $B C K / B C I / B$ - Algebras. BI- algebra is an algebra of type $(2,0)$ satisfying the following axioms:

1) $a \star a=0$.

2) $a \star(b \star a)=a$. for all $a, b \in X$.

In [1] a non -empty subset $\mathrm{S}$ of BI-algebra $\mathrm{X}$ is said to be a sub algebra of $\mathrm{X}$ if it is closed under the operation " $\star$ ", since $a \star a=0$, for all $a \in X$. It follows that $0 \in S$.

Suad Abdulaali Neamah and Ayat Abdulaadi Neamah in [15] initiated the idea of sub-implicative ideal of a $\mathrm{BH}$-algebra and deal with the relation ships among the ideal with their intersection, union of image of functions, and inverse function for subimplicative ideals of BH- algebra.

Sunshin ahn, and et al in [16] introduced BI-Ideals, normal subalgebras in BI-algebras and they obtain the quotient BI-algebra which is useful for the study of structures of BI-algebras.

The concept of fuzzy set which was introduced by Zadeh,L.A. in 18 provides a natural frame work for generalizing many of the concepts of general mathematics and topology. Karrar Dejaa,Mohamed in [12] initaited the idea of fuzzy $\lambda-$ ideal of BH-algebra is ordinary and fuzzy senses ,and give some properties of $\lambda$ - ideals,and $\mathrm{Hu}-$ sein Hadi Abbas and Suad Abd Neamah in 8 dealt with fuzzy implicative ideal of a BH-algebra and give some properties of fuzzy ideal and other types of fuzzy ideals and fuzzy subsets of a $\mathrm{BH}-$ algebra,and Gerima in 4 initiated basic ideals about fuzzy ideals and fuzzy filters on implication algebra.

Khosravishoar,S. in [13] revealed the idea of a fuzzy normal congruence on a group and the concepts of a fuzzy relation on a group; and Young Bae Jun, Roh,E.H., Chinju,and Hee sik Kim,seoul in [17] discussed on the fuzzification of B-sub algebras and 
some related properties of fuzzy B-algebras.

Kandil,A. ,and et al in 11 made contribution on separation and regularity axioms in fuzzy topology on fuzzy set,and some of its characterization and certain relation ship among them was discussed. Foster in 3 discussed basic ideas about fuzzy topological groups. The concepts of fuzzy BI-algebra is introduced by Gerima T. and Abdi O. in [5] and Gerima,T. and et al introduced ideals and filters on implication algebra in [6].

In this paper X represents BI-algebra unless otherwise mentioned.

\section{PRELIMINARIES}

In 1] A partially ordering $\leq$ on $\mathrm{X}$ can be defined by $a \leq b$ if and only if $a \star b=0$.

Proposition II..1 In [1] Let $X$ be a BI-algebra. Then the following hold:

1) $a \star 0=a$.

2) $0 \star a=0$.

3) $a \star b=(a \star b) \star b$.

4) If $b \star a=a$, for all $a, b \in X$, then $X=\{0\}$.

5) If $a \star(b \star c)=b \star(a \star c)$, for all $a, b \in X$, then $X=\{0\}$.

6) If $a \star b=c$, then $c \star b=c$ and $b \star c=b$.

7) If $(a \star b) \star(c \star d)=(a \star c) \star(b \star d)$, then $X=$ $\{0\}$, for all $a, b, c, d \in X$.

A BI-algebra $(X, \star, 0)$ is said to be right distributive [Left distributive] if for all $a, b, c \in X$, we have $(a \star b) \star c=(a \star c) \star(b \star c)[c \star(a \star b)=(c \star a) \star(c \star b)]$. In [1] a subset I of $\mathrm{X}$ is called an ideal of $\mathrm{X}$ if

1) $0 \in I$.

2) $a \star b \in I$ and $b \in I$ imply $a \in I$,for any $a, b \in X$.

An ideal $I$ is said to be proper ideal of $I \neq X$.

Definition II..2 $A$ non-empty subset $S$ of a $B I$ algebra $X$ is said to be a subalgebra if $a, b \in X$, then $a \star b \in S$.

Definition II..3 In [16] A non- empty subset $N$ of $X$ is said to be normal (or a normal subalgebra) if $(x \star a) \star(y \star b) \in N$, for any $x \star y, a \star b \in N$.

Proposition II..4 [16] Let $N$ be a normal sub algebra of $X$. Then $N$ is a sub algebra of $X$.

Example II..1 In [1] let $X=\{0, a, b, c\}$ be $a$ $B I$-algebra with the following table:

\begin{tabular}{|c|c|c|c|c|}
\hline$\star$ & 0 & $a$ & $b$ & $c$ \\
\hline 0 & 0 & 0 & 0 & 0 \\
\hline$a$ & $a$ & 0 & 0 & 0 \\
\hline$b$ & $b$ & 0 & 0 & $b$ \\
\hline$c$ & $c$ & 0 & $c$ & 0 \\
\hline
\end{tabular}

Then $\{0, a, b\}$ is a sub algebra of $X$ but not normal, since $c \star c=0, b \star c=b \in\{0, a, b\}$, but $(c \star b) \star(c \star c)=$ $(c \star b) \star 0=c \star b=c \notin\{0, a, b\}$.

Lemma II..5 In [16] let $N$ be a normal sub algebra of $X$. If $a \star b \in N$, for all $a, b \in X$, then $b \star a \in N$.

Definition II..6 [16] Let I be an ideal of X. Then $I$ is called a normal ideal of $X$ if it is normal.

Proposition II..7 [16] Let I be a normal ideal of $X$. Then $I$ is a sub algebra of $X$.

Definition II..8 17 fuzzy subset $\mu$ in $X$ is called a fuzzy $B$ - subalgebra if it satisfies the inequality $\mu(a \star b) \geq$ $\min \{\mu(a), \mu(b)$, for all $a, b \in X\}$.

In [12] A fuzzy subset $\mu$ of a $\mathrm{BH}$-algebra $\mathrm{X}$ is said to be a fuzzy ideal if and only if

1) $\mu(0) \geq \mu(a)$, for all $a \in X$.

2) $\mu(a) \geq \min \{\mu(a \star b), \mu(b)\}$, for all $a, b \in X$.

Let $I=[0,1]$ and $I^{X}=\{\mu: X \rightarrow I\}$. Then the family of all fuzzy subsets of $A=$ $\{<x, \mu(x)>: x \in X\}$ denoted by $F_{A}$. That is $F_{A}=\left\{B \in I^{X}: B \subseteq A\right\}$.

If $A, B \in I^{X}$ and $B(x) \subseteq A(x)$,for all $x \in X$, then $\mathrm{B}$ is said to be a fuzzy subset of $\mathrm{A}$ and denoted by $B \subseteq A$.

The set $S(\mu)=\{x \in X: \mu(x)>0\}$ is said to be the supper set of $\mu$.

Lemma II..2 [11] Let $U, V \in F_{A}$ and $\left\{V_{i}, i \in J\right\} \subset F_{A}$. Then

1) $S(U \cap V)=S(U) \cap S(V)$.

2) $S\left(\cup_{i \in J} V_{i}\right)=\cup_{i \in J} S\left(V_{i}\right)$.

In[11] $A=\{\langle x, \mu(x)\rangle \quad x \in X\}$ be a fuzzy subset of X. A collection $\sigma$ of fuzzy subsets of A. That is $\sigma \in F_{A}$ satisfying the following condition:

1) $0, A \in \sigma$.

2) $U, V \in \sigma$ imply $U \cap V \in \sigma$.

3) $\left\{V_{i}, i \in J\right\} \subset \sigma$ implies $\cup_{i \in J} V_{i} \in \sigma$ is called a fuzzy topology on A. 
The pair $(A, \sigma)$ is called a fuzzy topological space , members of $\sigma$ called a fuzzy open sets and their complements are called fuzzy closed sets of $(A, \sigma)$.

Definition II..9 [5] Let $(X, \star, 0)$ be a BI- algebra. Then the fuzzy subset $\mu$ of $X$ is called a fuzzy $B I-$ sub algebra if $\mu(a \star b) \geq \mu(a) \wedge \mu(b)$, Where $\mu(a) \wedge \mu(b)=\inf \{\mu(a), \mu(b)\} \quad$ for all $a, b \in X$.

\section{RESULTS}

A. Homomorphisms in fuzzy BI- algebras

Definition III..1 [16] Let $X$ and $Y$ be $B I$ algebras. Then $f: X \rightarrow Y$ defined by $f(a \star b)=$ $f(a) \star f(b)$, for all $a, b \in X$ is said to be a homomorphism in BI-algebra.

Definition III..2 Let $X$ and $Y$ be any two sets. $\mu$ be any fuzzy subset of $X$, and let $f$ : $X \rightarrow Y$ be any function. The set $f^{-1}(b)=$ $\{a \in X \mid f(a)=f(b), \forall b \in Y\}$. The set $B$ in $Y$ defined by $B(y)=\left\{\begin{array}{ll}\operatorname{Sup}(\mu(a)) & \text { if } f^{-1}(b) \neq \emptyset \\ 0 & \text { otherwise }\end{array}\right.$ for all $b \in Y$ is called the image of $\mu$ under $f$ and is denoted by $f(\mu)$.

Definition III..3 Let $X$ and $Y$ be any two sets, $f$ : $X \rightarrow Y$ be any function and $B$ be any fuzzy set in $f(\mu)$. The fuzzy subset $\mu$ in $X$ defined by $\mu(a)=B(f(a))$, for all $a \in X$ is called the image of $B$ under $f$ and is denoted by $f^{-1}(b)$. That is $f^{-1}(B)(a)=B f(a)$.

Proposition III..4 Let $f: X \rightarrow Y$ be a $B I-$ epimorphism. If $\mu$ is a fuzzy ideal of $X$, then $f(\mu)$ is a fuzzy ideal of $Y$.

Proof. Let $f: X \rightarrow Y$ be a $B I-$ epimorphism and let $\mu$ be a fuzzy ideal of $\mathrm{X}$. Then

$$
\text { 1) } \begin{aligned}
& f(\mu)(0)= \begin{cases}\operatorname{Sup}(\mu(0)) & \text { if } f^{-1}(0) \neq \emptyset \\
0 & \text { otherwise }\end{cases} \\
& \geq \begin{cases}\operatorname{Sup}\left(\mu(a)_{a \in f^{-1}(0)}\right) & \text { if } f^{-1}(0) \neq \emptyset \\
0 & \text { otherwise }\end{cases} \\
&=f(\mu)(b)
\end{aligned}
$$

Imply that $f(\mu)(0) \geq f(\mu)(b)$, for all $b \in Y$

$=f(\mu)(b), f^{-1}(b) \neq \emptyset$.

2)

$$
\begin{aligned}
& f(\mu)(c \quad \star \quad d)) \\
& \begin{cases}S u p(\mu(a \star b)) & \text { if } f^{-1}(c \star d) \neq \emptyset \\
0 & \text { otherwise }\end{cases} \\
& \geq \begin{cases}\operatorname{Sup}(\mu(a \star b)) \wedge \sup (\mu(b)) & \text { if } f^{-1}(c \star d) \neq \emptyset \\
0 & \text { otherwise }\end{cases} \\
& =f(\mu)(c \quad d) \\
& f(\mu)(d), \text { if } a \star b \in f^{-1}(c \star d), b \in f^{-1}(d) .
\end{aligned}
$$

3) Left for reader.
Proposition III..5 Let $f: X \rightarrow Y$ be a $B I-$ homomorphism. If $\mu$ is a fuzzy ideal of $Y$, then $f^{-1}(\mu)$ is a fuzzy ideal of $X$.

Proof. Let $f: X \rightarrow Y$ be $B I-$ homomorphism and let $\mu$ be a fuzzy $B I-$ ideal of $\mathrm{X}$. Then

1) $f^{-1}(\mu)(0)=\mu(f(0))$

$$
\begin{aligned}
& =\mu(f(a \star a)) \\
& =\mu(f(a) \star f(a)) \\
& \geq \mu(f(a)) \wedge \mu(f(a)) \\
& =f^{-1}(\mu)(a) \wedge f^{-1}(\mu)(a)=f^{-1}(\mu)(a) .
\end{aligned}
$$

Hence $f^{-1}(\mu)(0) \geq f^{-1}(\mu)(a)$,for all $a \in X$.

2) $f^{-1}(\mu(a))=\mu(f(a)) \geq \mu(f(a \star b)) \wedge \mu(f(b))$ $=f^{-1}(\mu(a \star b)) \wedge f^{-1}(\mu(b))$.

Hence $f^{-1}(\mu(a)) \geq f^{-1}\left(\mu(a \star b) \wedge f^{-1}(\mu(b))\right.$.

3) $f^{-1}(\mu(a \star b))=\mu(f(a \star b))$

$=\mu(f(a) \star f(b))$ sncefishomomorphism.

$\geq \mu(f(a)) \wedge \mu(f(b))=f^{-1}(\mu(a)) \wedge f^{-1}(\mu(b))$.

Hence $f^{-1}(\mu(a \star b)) \geq f^{-1}(\mu(a)) \wedge$ $f^{-1}(\mu(b))$, for all $a, b \in X$.

Hence $f^{-1}(\mu)$ is a fuzzy ideal of $\mathrm{X}$.

B. Cartesian product in fuzzy ideal of BIalgebras

Definition III..6 Let $\lambda$ and $\mu$ be a fuzzy ideals of a BI-algebra $X$. Then the Cartesian product of $\lambda$ and $\mu, \lambda \times \mu: X \times X \rightarrow[0,1]$ defined by $\lambda \times \mu(a, b)=$ $\lambda(a) \wedge \mu(b)$,forall $a, b \in X$. Where $\lambda(a) \wedge \mu(b)=$ inf $\{\lambda(a), \mu(b)\}$, for all $a, b \in X$.

Theorem III..7 If $\lambda$ and $\mu$ are fuzzy ideal of $B I$-algebra,then $\lambda \times \mu$ is a fuzzy ideal of BI-algebra.

Proof. Let $\lambda$ and $\mu$ be a fuzzy ideals of $B I-$ algebra X. Then

1) $(\lambda \times \mu)(0,0)=\lambda(0) \wedge \mu(0)$

$\geq \lambda(a) \wedge \mu(b)=(\lambda \times \mu)(a, b)$, for all $a, b \in X$.

Hence $(\lambda \wedge \mu)(0,0) \geq(\lambda \times$ $\mu)(a, b)$,for all $a, b \in X$.

2) $(\lambda \times \mu)(a, a)=\lambda(a) \wedge \mu(a)$ $\geq(\lambda(a \star b) \wedge \lambda(b)) \wedge(\mu(a \star b) \wedge \mu(b))$

$=(\lambda(a \star b) \wedge \mu(a \star b)) \wedge(\lambda(b) \wedge \mu(b))$

$=(\lambda \times \mu)(a \star b) \wedge(\lambda \times \mu)(b)$.

Hence $(\lambda \times \mu)(a, a) \geq(\lambda \times \mu)(a \star b) \wedge(\lambda \times$ $\mu)(b)$,for all $a, b \in X$.

3) $(\lambda \times \mu)(a \star b, c \star d))=\lambda(a \star b) \wedge \mu(c \star d)$

$\geq(\lambda(a) \wedge \lambda(b)) \wedge(\mu(c) \wedge \mu(d))$

$=(\lambda(a) \wedge \mu(c)) \wedge(\lambda(b) \wedge \mu(d))$

$=(\lambda \times \mu)(a, c) \wedge(\lambda \times \mu)(b, d)$.

Hence $(\lambda \times \mu)(a \star b) \geq(\lambda \times \mu)(a, c) \wedge(\lambda \times$ $\mu)(b, d)$,forall $a, b, c, d \in X$.

Therefore $\lambda \times \mu$ is a fuzzy ideal of $B I-$ algebra. 
Proposition III..8 Let $\lambda$ and $\mu$ be a fuzzy ideal of BI-algebra and let $\lambda \times \mu$ be a fuzzy ideal of $B I$-algebra. Then $\lambda \times \mu$ is a fuzzy subalgebra of $B I$-algebra $X$.

\section{Fuzzy topology on BI- algebra}

Definition III..9 Let $T$ be a fuzzy topology on $X$, and let $\mu$ be a fuzzy BI-algebra of $X$ with induced topology $T_{\mu}$. Then $T$ is called a fuzzy topological BI- algebra of $X$ if for each $a \in X$ the mapping $Q_{a}:\left(\mu, T_{\mu}\right) \rightarrow\left(\mu, T_{\mu}\right)$ is relatively fuzzy continuous.

Theorem III..10 Let $X$ and $Y$ be two $B I$-algebras, $f: X \rightarrow Y$ be a BI- homomorphism. Let $T$ and $S$ be the fuzzy topology on $X$, and $Y$ respectively such that $T=f^{-1}(S)$, and let $A$ be any fuzzy topological BI-algebra of $Y$ with membership function $\mu_{A}$, where $\mu$ is a fuzzy $B I$ - algebra. Then $f^{-1}(A)$ is a fuzzy topological BI-algebra of $X$ with membership function $\mu_{f^{-1}(A)}$.

Proof. For each $a \in X$, the mapping $Q_{a}$ : $\left(f^{-1}(A), T_{f^{-1}(A)}\right) \rightarrow\left(f^{-1}(A), T_{f^{-1}(A)}\right)$ is relatively fuzzy continuous.

Let $\mathrm{U}$ be any open fuzzy set in $T_{f^{-1}(A)}$ on $f^{-1}(A)$. Since $\mathrm{f}$ is a fuzzy continuous mapping from $(X, T)$ into $(Y, S)$ by lemma 2.2 follows that $\mathrm{f}$ is relatively fuzzy continuous mapping of $\left(f^{-1}(A), T_{f^{-1}(A)}\right)$ into $\left(A, S_{A}\right)$. There exists an open fuzzy set $v \in S_{A}$ such that $f^{-1}(V)=U$. The membership function of $Q_{a}^{-1}(U)$ is given by $\mu_{Q_{a}^{-1}(U)}(b)=\mu_{U}\left(Q_{a}(b)\right)$

$=\mu_{U}(b \star a)=\mu_{f-1(V)}(b \star a)=\mu_{V}(f(b \star a))$

$=\mu_{V}(f(b) \star f(a))$. Since $\mathrm{A}$ is a fuzzy topological $B I$ - sub algebra of $\mathrm{Y}$, the mapping

$Q_{a}:\left(A, S_{A}\right) \rightarrow\left(A, q_{A}\right)$ is relatively fuzzy continuous for each $b \in Y$.

Hence $\mu_{Q_{a}^{-1}(U)}(b)=\mu_{V}(f(b) \star f(a))$

$=\mu_{V}\left(R_{f(a)}(f(b))\right.$

$=\mu_{R_{f(a)}^{-1}}(V)(f(b))=\mu_{f_{-1}\left(R_{f(a)}^{-1}(V)\right.}(b)$ which implies that $Q_{a}^{-1}(U)=f^{-1}\left(R_{f(a)}^{-1}(V)\right)$.

Therefore $Q_{a}^{-1}(U) \cap f^{-1}(A)=f^{-1}\left(R_{f(a)}^{-1}(V)\right) \cap$ $f^{-1}(A)$ is open in the relative fuzzy topology $f^{-1}(A)$.

Theorem III..11 Given BI-algebras $X$ and $Y$ and let $f: X \rightarrow Y$ be Bi- epimorphism, let $T$ be the fuzzy topology on $X$, and $S$ be the fuzzy topology on $Y$ such that $f(T)=S$. Let $A$ be a fuzzy topological BI-algebra of $X$. If the membership function $\mu_{A}$ of $A$ is an $f$ - invariant, then $f(A)$ is a fuzzy topological BI-sub algebra of $Y$.

Proof. We have to show the mapping $Q_{a}$ : $\left(f(A), S_{f(A)}\right) \rightarrow\left(f(A), S_{f(A)}\right)$ is relatively fuzzy continuous for all $b \in Y$.

Since $U \in T_{A}$ there exists $U^{\prime} \in T$ such that
$U=U^{\prime} \cap A$, by $f$-invariant of $\mu_{A}$, we have $f(U)=f\left(U^{\prime} \cap A\right)=f\left(U^{\prime}\right) \cap f(A) \in S_{f(A)}$.

Hence $\mathrm{f}$ is a relatively fuzzy open mapping in $S_{f(A)}$. Let $V^{\prime}$ be an open fuzzy set in $S_{f(A)}$. For any $b \in Y$ by hypothesis there exist $a \in X$ such that $f(a)=b$. Thus $\mu_{f^{-1}\left(Q_{a}^{-1}\left(V^{\prime}\right)\right.}(c)=\mu\left(Q_{f(a)}\left(V^{\prime}\right)(c)=\right.$ $\mu_{V^{\prime}}\left(Q_{f(a)}(f(c))\right.$

$=\mu_{V^{\prime}}(f(c) \star f(a))=\mu_{V^{\prime}}(f(c \star a))$

$=\mu_{f^{-1}\left(V^{\prime}\right)}(c \star a)=\mu_{f^{-1}\left(V^{\prime}\right)}\left(Q_{a}(c)\right)=$ $\mu_{Q_{a}^{-1}\left(f^{-1}\left(V^{\prime}\right)\right.}(c)$.

Hence $f^{-1}\left(Q_{a}^{-1}\left(V^{\prime}\right)=Q_{a}^{-1}\left(f^{-1}\left(V^{\prime}\right)\right)\right.$. But by hypothesis $Q_{a}$ is a relatively fuzzy continuous mapping from $\left(A, T_{A}\right)$ to $\left(A, T_{A}\right)$, and $\mathrm{f}$ is a relatively fuzzy continuous mapping from $\left(A, T_{A}\right)$ $\operatorname{to}\left(f(A), S_{f(A)}\right)$.

Therefore $f^{-1}\left(Q_{a}^{-1}\left(V^{\prime}\right)\right) \cap A=Q_{a}^{-1}\left(f^{-1}\left(V^{\prime}\right)\right) \cap A$ is open in $T_{A}$.

Since $\mathrm{f}$ is relatively open , then $f\left(f^{-1}\left(Q_{a}^{-1}\left(V^{\prime}\right)\right) \cap\right.$ $A)=f\left(f^{-1}\left(Q_{a}^{-1}\left(V^{\prime}\right)\right) \cap f(A)\right.$

$=Q_{a}^{-1}\left(V^{\prime}\right) \cap f(A)$ is open in $S_{f(A)}$. Which completes the proof.

\section{Conclusions}

In this paper. the concepts of homomorphism in fuzzy BI-algebras are discussed, and also basic properties of homomorphisms are investigated.

The cartesian product in fuzzy ideals of BI-algebras has been investigated with related properties; The concepts of fuzzy topology on BI-algebra is elaborated. As a future work it is possible to extend to coding BI-algebra,fuzzy dot product of ideals of BI-algebras.

\section{ACKNOWLEDGMENTS}

This research is supported by Wollo University research center, and the authors would like to give great thanks to Wollo University research center and the referees for their genuine comment for improvement of the manuscript .

\section{REFERENCES}

[1] Arsham Boruman Saeid, Hee Sik Kim and Akbar Rezae, On BI- Algebras, An.st.Univ.Oridios constanta, vol. 25(1),2017,pp.177-194,Doi:10.1515 — auom2017-0014.

[2] Boezooel,R.A.,Khosravishoar,S.,Implication algebras are equivalent to the dual implicative BCK-Algebras, Scientia Mathematicae Japonicae, on line ,e-2006, PP.371-373.

[3] Foster,D.H.,Fuzzy topological groups , J.Math.Anal.Appl.,67(1979),549-564.

[4] Gerima T., Fuzzy Ideals and Fuzzy filters in an implication algebra, On communication for publication,2020(9 page). 
[5] Gerima T., and Abdi O., On Fuzzy BI-Algebra, On Communication ,2021(11 pages)

[6] Gerima ,T., Endris,Y. and Fasil,G., Ideals and Filters on implication algebras, Advances in Mathematics:Scientific Journals, Vol.10(2021), No. 3, 1167-1174, ISSN : 18578365(printed);1877-8435(electronic).

[7] Huang,Y.S., BCI-algebra, Science press, China,2006,ISBn:9787-03-015411-8.

[8] Husein Hadi Abbas , and Suad Abd Neamah, On the fuzzy implicative ideals of a $\mathrm{BH}-$ algebras, Vol. 12(4),(Aug.2016).PP.59-70, Doi:10.9790/5728-1204055970.

[9] Imai, Y., and Iseki, K., On axioms systems of propositional calculi, Xiv proc.Japan Academy,Vol. 42(1966), pp.19-22.

[10] , Jun,Y.B.,Roh, E.H.,and Kim,H.S., Sci.math.Jpn.1/1998, On BH-algebras,pp.347354.

[11] Kandil,A., Saleh,S., and Myakout,M., Fuzzy topology on fuzzy sets; Regularity and separation axioms, American Academic and Scholarly Research Journals,vol. 4(2), March 2012.

[12] Karrar Dejaa Mohammed. A fuzzy $\lambda$ - ideal of a BH-algebra, Journal of Kufa for Mathematics and Computer, Vol. 4 ,No.2,June ,2017, pp. 2733.

[13] Khosravishoar,S., Fuzzy normal congruences and fuzzy coset relations on Groups,International Journal of pure and applied Mathematics ,Vol.115, No. 2,2017, pp.211-224, Doi: 10.12732/ijpam.v115i2.2.

[14] Neggers,J., and Kim,H.S., On Balgebras,Math.Vensik, Vol.54(2002),pp. 21-29.

[15] Sud Abdulaali Neamah, and Ayat Abdulaali Neamah, On the sub implicative ideals of a BH-algebra, Journal of University of Kerbala, vol.16,No. 1, 2018.

[16] Sunshin Ahn, Jungmiko, and Borumano Said, A., On Ideals of BI-algebras,J. Indones.math. Soc., vol. 25, No.(2019), pp.24-34.

[17] Young Bae Jun, Roh,E,H.,Chinju, and Hee Sik Kim, Seoul , On fuzzy B-algebras, Czechoslovak Mathematical Journal, Vol. 52(127),2002,pp. 375-384.

[18] Zadeh,L.A. Fuzzy sets, Inform and Control,8(1965),338-353.

Creative Commons Attribution License 4.0 (Attribution 4.0 International, CC BY 4.0)

This article is published under the terms of the Creative Commons Attribution License 4.0 